\title{
HEAT KERNELS FOR A FAMILY OF GRUSHIN OPERATORS*
}

\author{
DER-CHEN CHANG ${ }^{\dagger}$ AND YUTIAN LI ${ }^{\ddagger}$
}

\section{Dedicated to Professor Stephen S. T. Yau on the occasion of his 60th birthday}

Abstract. We construct the heat kernel for the second-order operator $\Delta_{\mathbf{X}}=\frac{1}{2} \sum_{k=1}^{n}\left(\frac{\partial}{\partial x_{k}}\right)^{2}+$ $\frac{1}{2} \sum_{k=1}^{n}\left(x_{k}^{m_{k}} \frac{\partial}{\partial y_{k}}\right)^{2}$ with $m_{k} \in \mathbf{N}$, which is a degenerate elliptic operator. Obviously, this operator is closed related to the Grushin operator $L_{G}=\frac{1}{2}\left(\frac{\partial}{\partial x}\right)^{2}+\frac{1}{2}\left(x^{m} \frac{\partial}{\partial y}\right)^{2}$ with $m \in \mathbf{N}$. In this paper, we fist give a complete description of the geometry induced by the operator $L_{G}$. More precisely, given any two points in the space, the number of geodesics and the lengths of the geodesics are calculated. Then we find modified complex action functions and show that the critical values of this function will recover the lengths of the corresponding geodesics. We also find the volume element by solving a generalized transport equation. Finally, the formula for the heat kernel of the diffusion operator $\frac{\partial}{\partial t}-\Delta_{\mathbf{X}}$ is obtained. The formula involves an integral of a product between the volume function and an exponential term. ment.

Key words. Grushin operators, subRiemannian geometry, geodesics, heat kernel, volume ele-

AMS subject classifications. Primary 53C17, 53C22; Secondary 35H20.

1. Introduction. In this paper, we want to find the heat kernels for operators $L_{j}, j=1,2,3,4$, where

$$
\begin{aligned}
& L_{1}=\frac{1}{2} \sum_{k=1}^{n}\left(\frac{\partial}{\partial x_{k}}\right)^{2}+\frac{1}{2} \sum_{k=1}^{n}\left(x_{k}^{m_{k}} \frac{\partial}{\partial y_{k}}\right)^{2} \\
& L_{2}=\frac{1}{2}\left(\frac{\partial}{\partial x}\right)^{2}+\frac{1}{2} \sum_{k=1}^{n}\left(x^{m} \frac{\partial}{\partial y_{k}}\right)^{2} \\
& L_{3}=\frac{1}{2} \sum_{k=1}^{n}\left(\frac{\partial}{\partial x_{k}}\right)^{2}+\frac{1}{2} \sum_{k=1}^{n}\left(x_{k}^{m_{k}} \frac{\partial}{\partial y}\right)^{2}
\end{aligned}
$$

and

$$
L_{4}=\frac{1}{2} \sum_{k=1}^{n}\left(\frac{\partial}{\partial x_{k}}\right)^{2}+\frac{1}{2} \sum_{k=1}^{n}\left(x_{k}^{m_{k}} \frac{\partial}{\partial y_{k}}\right)^{2}+\frac{1}{2}\left(\frac{\partial}{\partial x_{n+1}}\right)^{2} .
$$

Here $m_{k}=1,2, \ldots$ and $k=1, \ldots, n$.

All these operators can be realized as sums of squares of vector fields which are elliptic operators except when $\left\{x_{k}=0, k=1, \ldots, n\right\}$. We refer this set as the missing directions. All these operators are closely related to the Grushin operator

$$
L_{G}=\frac{1}{2}\left(X_{1}^{2}+X_{2}^{2}\right)=\frac{1}{2}\left(\frac{\partial}{\partial x}\right)^{2}+\frac{x^{2 m}}{2}\left(\frac{\partial}{\partial y}\right)^{2},
$$

*Received September 23, 2012; accepted for publication December 14, 2012.

$\dagger$ Department of Mathematics and Department of Computer Science, Georgetown University, Washington D.C. 20057, USA (chang@georgetown.edu); Department of Mathematics, Fu Jen Catholic University, Taipei 242, Taiwan, ROC. The first author is partially supported by an NSF grant DMS-1203845 and Hong Kong RGC competitive earmarked research grant \#601410.

$\ddagger$ Institute of Computational and Theoretical Studies, and Department of Mathematics, Hong Kong Baptist University, Kowloon, Hong Kong (yutianli@hkbu.edu.hk). 
where $X_{1}=\frac{\partial}{\partial x}$ and $X_{2}=x^{m} \frac{\partial}{\partial y}$ with $m \in \mathbf{N}$. The operator $L_{1}$ can be realized as a product of Grushin operators defined on the product space $\mathbf{R}^{2} \times \cdots \times \mathbf{R}^{2}$. Hence, it has $n$ missing directions. The operator $L_{2}$ is a Grushin operator defined on $\mathbf{R}^{n+1}$ with $n$ missing directions. The operator $L_{3}$ is a Grushin operator defined on $\mathbf{R}^{n+1}$ with only 1 missing direction. Finally, the operator $L_{4}$ is a generalized Baouendi-Goulaouic operator defined on $\mathbf{R}^{2 n+1}$ which is a sum of Grushin and Laplace operators.

We shall recall the heat kernel for the Laplace-Beltrami operator

$$
\Delta=-\frac{1}{2} \sum_{j=1}^{n} X_{j}^{*} X_{j}=\frac{1}{2} \sum_{j=1}^{n} X_{j}^{2}+\cdots .
$$

Here $X_{1}, \ldots, X_{n}$ represent $n$ linearly independent vector fields on an $n$-dimensional manifold $\mathcal{M}_{n}$. The heat kernel for $\Delta$, at least locally, takes the form,

$$
\mathcal{P}_{t}\left(\mathbf{x}, \mathbf{x}_{0}\right)=\frac{1}{(2 \pi t)^{\frac{n}{2}}} e^{-\frac{d^{2}\left(\mathbf{x}, \mathbf{x}_{0}\right)}{2 t}}\left(a_{0}+a_{1} t+a_{2} t^{2}+\cdots\right)
$$

The $a_{j}$ 's are functions of $\mathbf{x}$ and $\mathbf{x}_{0}$, and $d\left(\mathbf{x}, \mathbf{x}_{0}\right)$ denotes the Riemannian distance between $\mathbf{x}_{0}$ and $\mathbf{x}_{1}$. Note that

$$
\frac{\partial}{\partial t}\left(\frac{d^{2}\left(\mathbf{x}, \mathbf{x}_{0}\right)}{2 t}\right)+\frac{1}{2} \sum_{j=1}^{n}\left(X_{j} \frac{d^{2}\left(\mathbf{x}, \mathbf{x}_{0}\right)}{2 t}\right)^{2}=0
$$

i.e., $\frac{d^{2}\left(\mathbf{x}, \mathbf{x}_{0}\right)}{2 t}$ is a solution of the Hamilton-Jacobi equation.

We are interested in finding the solving kernels for the operators $\frac{\partial}{\partial t}-L_{j}, j=$ $1,2,3,4$. It is reasonable to expect the kernel have the form:

$$
\mathcal{P}_{t}\left(\mathbf{x}, \mathbf{x}_{0}\right)=\frac{c}{t^{\alpha}} e^{-h_{j}\left(\mathbf{x}, \mathbf{x}_{0}\right)} \quad \text { for some suitable } \alpha,
$$

where the modified complex action function $h_{j}\left(\mathbf{x}, \mathbf{x}_{0}\right)$ plays the role of $\frac{d^{2}\left(\mathbf{x}, \mathbf{x}_{0}\right)}{2 t}$ and satisfies the Hamilton-Jacobi equation

$$
\frac{\partial h_{j}}{\partial t}+H\left(x, y, \frac{\partial h_{j}}{\partial x}, \frac{\partial h_{j}}{\partial y}\right)=0, \quad j=1,2,3,4 .
$$

Given the vector fields $X_{1}, \ldots, X_{\ell}$ in an $n$-dimensional manifold $\mathcal{M}_{n}$ with $\ell \leq$ $n$, assume that $\mathbf{X}=\left\{X_{1}, \ldots, X_{\ell}\right\}$ satisfies the bracket generating condition: "the horizontal vector fields $\mathbf{X}$ and their brackets span $T \mathcal{M}_{n}$ ". Based on this assumption, Chow [16] proved the following celebrated theorem in 1939.

TheOREM 1. Given any two points $A, B \in \mathcal{M}_{n}$, there is a piecewise $C^{1}$-horizontal curve $\gamma:[0, \tau] \rightarrow \mathcal{M}_{n}$ :

$$
\gamma(0)=A, \quad \gamma(\tau)=B
$$

and

$$
\dot{\gamma}(s)=\sum_{k=1}^{\ell} a_{k}(s) X_{k} \quad \text { almost everywhere. }
$$

Now we may define a geometry on on the manifold $\mathcal{M}_{n}$. A sub-Riemannian structure over a manifold $\mathcal{M}_{n}$ is a pair $(\mathcal{H},\langle\cdot, \cdot\rangle)$, where $\mathcal{H}$ is a bracket generating 
distribution and $\langle\cdot, \cdot\rangle$ a fibre inner product defined on $\mathcal{H}$. Now by Chow's theorem, given two points $A$ and $B$ in $\mathcal{M}_{n}$, one may measure the length of the horizontal curve $\gamma$ by

$$
\ell(\gamma):=\int_{0}^{\tau} \sqrt{\langle\dot{\gamma}(s), \dot{\gamma}(s)\rangle} d s
$$

The shortest length

$$
d_{c c}(A, B):=\inf \ell(\gamma)
$$

is called a Carnot-Carathéodory distance (see Calin and Chang [7], Gromov [23] and Strichartz [28]) between $A, B \in \mathcal{M}_{n}$ where the infimum is taken over all absolutely continuous horizontal curves joining $A$ and $B$.

Such subRiemannian geometry is useful in understanding the fundamental solution and heat kernel for the corresponding operator $\Delta_{\mathbf{X}}=\frac{1}{2} \sum_{k=1}^{\ell} X_{j}^{2}$; see e.g. $[2,3,4,5]$. As a consequence, one can recover the well-known Hörmander theorem [25]. $\Delta_{\mathbf{X}}$ is hypoelliptic, i.e., if $\Delta_{\mathbf{X}} u=f \in C^{\infty}$, then $u \in C^{\infty}$.

Set

$$
X_{j}=\sum_{k=1}^{n} a_{j k}(x) \frac{\partial}{\partial x_{k}}, \quad j=1, \ldots, \ell .
$$

Then

$$
H=\frac{1}{2} \sum_{j=1}^{\ell}\left(\sum_{k=1}^{n} a_{j k}(x) \xi_{k}\right)^{2}
$$

is the Hamiltonian function on the cotangent bundle $T^{*} \mathcal{M}_{n}$. A bicharacteristic curve $(\mathbf{x}(s), \xi(s)) \in T^{*} \mathcal{M}_{n}$ is a solution of the Hamilton's system:

$$
\dot{x}_{k}(s)=H_{\xi_{k}}, \quad \dot{\xi}_{k}(s)=-H_{x_{k}},
$$

with boundary conditions

$$
x_{k}(0)=x_{k 0}, \quad x_{k}(\tau)=x_{k}, \quad k=1, \ldots, n,
$$

for given points $\mathbf{x}_{0}, \mathbf{x} \in \mathcal{M}_{n}$. The projection $\mathbf{x}(s)$ of a bicharacteristic curve on $\mathcal{M}_{n}$ is a geodesic.

In the first part of this paper, we survey the geometry which is induced by the famous Grushin's operator (see [24]):

$$
\Delta_{m}=\frac{1}{2} \frac{\partial^{2}}{\partial x^{2}}+\frac{1}{2} x^{2 m} \frac{\partial^{2}}{\partial y^{2}}=\frac{1}{2}\left(X_{1}^{2}+X_{2}^{2}\right),
$$

where $m \in \mathbf{N}$ with

$$
X_{1}=\frac{\partial}{\partial x}, \quad X_{2}=x^{m} \frac{\partial}{\partial y}
$$

Since it is easy to see that $\Delta_{m}=\frac{1}{2} \frac{\partial^{2}}{\partial x^{2}}$ along the $y$-axis, then it is non-elliptic. However, the first $m$ Lie brackets are:

$$
\left[X_{1}, X_{2}\right]=m x^{m-1} \frac{\partial}{\partial y}
$$




$$
\begin{gathered}
{\left[X_{1},\left[X_{1}, X_{2}\right]\right]=m(m-1) x^{m-2} \frac{\partial}{\partial y}} \\
\cdots \\
{\left[X_{1},\left[X_{1}, \cdots,\left[X_{1}, X_{2}\right]\right] \cdots\right]=m ! \frac{\partial}{\partial y} .}
\end{gathered}
$$

Hence $\left\{X_{1}, X_{2}\right\}$ satisfies the bracket generating condition.

In order to construct the heat kernel for $\Delta_{m}$, one needs to derive a modified action function $f$ which plays the same role as $d^{2}\left(\mathbf{x}, \mathbf{x}_{0}\right)$ in the Riemannian case. Hence, we need to obtain information on all "geodesics" connecting any two points in the Grushin plane.

When $m=1$, the geometry induced by the step 2 Grushin operator $\Delta_{1}=\frac{1}{2}\left(\frac{\partial^{2}}{\partial x^{2}}+\right.$ $\left.x^{2} \frac{\partial^{2}}{\partial y^{2}}\right)$ has been studied in detail in Calin et al. [8, 10] and Chang et al. [13, 14]. They obtained all geodesics between any two points in the Grushin plane. When $m=2$, Calin and Chang [6] studied the geometry induced by the step 3 Grushin operator $\Delta_{2}=\frac{1}{2}\left(\frac{\partial^{2}}{\partial x^{2}}+x^{4} \frac{\partial^{2}}{\partial y^{2}}\right)$. They obtained all geodesics which connect points on the $y$-axis with any other points in the Grushin plane by careful analysis of Jacobi elliptic functions.

2. Geodesics. Fix $m \in \mathbf{N}$. The Hamiltonian of the Grushin operator $\Delta_{m}$ is

$$
H(x, y, \xi, \eta)=\frac{1}{2}\left(\xi^{2}+x^{2 m} \eta^{2}\right)
$$

where $(\xi, \eta)$ are variables dual to $(x, y)$.

The normal geodesics between the points $P\left(x_{0}, y_{0}\right)$ and $Q\left(x_{1}, y_{1}\right)$ are the projections onto the $(x, y)$-plane of the solutions to the Hamiltonian system

$$
\left\{\begin{array}{l}
\dot{x}=H_{\xi}=\xi, \quad \dot{y}=H_{\eta}=\eta x^{2 m} \\
\dot{\xi}=-H_{x}=-m \eta^{2} x^{2 m-1}, \quad \dot{\eta}=-H_{y}=0
\end{array}\right.
$$

with the boundary conditions

$$
x(0)=x_{0}, \quad y(0)=y_{0}, \quad x(1)=x_{1}, \quad y(1)=y_{1} .
$$

The Hamiltonian system is invariant with respect to the symmetries

$$
(x, y ; \eta) \rightarrow(-x, y ; \eta), \quad(x, y ; \eta) \rightarrow(x,-y ;-\eta),
$$

so we may restrict our attention to $x_{1} \geq 0, y_{1}-y_{0} \geq 0, \eta \geq 0$, without loss of generality.

We note that $\dot{\eta}=0$ implies that $\eta$ is a constant which is the Lagrange multiplier. Set

$$
V(x)=\frac{1}{2} \eta^{2} x^{2 m}
$$

then (2.2) yields

$$
\ddot{x}=-V^{\prime}(x),
$$


which takes the form of the law of conservation of energy

$$
\frac{1}{2} \dot{x}^{2}+V(x)=E \quad \Leftrightarrow \quad \dot{x}^{2}+\eta^{2} x^{2 m}=2 E:=\eta^{2} A^{2 m},
$$

where $E$ is a constant, the total energy, which depends on the boundary conditions $x_{0}, y_{0}, x_{1}, y_{1}$ and the constant $\eta ; A$ is the amplitude of $x$. From equations (2.2) and $(2.5)$, one has

$$
\dot{x}^{2}+\frac{\dot{y}^{2}}{x^{2 m}}=\dot{x}^{2}+\eta^{2} x^{2 m}=\eta^{2} A^{2 m} .
$$

Let

$$
\gamma(s)=(x(s), y(s)):[0,1] \rightarrow \mathbb{R}^{2}
$$

be a horizontal curve. Then

$$
\frac{d}{d s}:=\dot{x} \frac{\partial}{\partial x}+\dot{y} \frac{\partial}{\partial y}=\alpha X_{1}+\beta X_{2}=\alpha \frac{\partial}{\partial x}+\beta x^{m} \frac{\partial}{\partial y} .
$$

The Carnot-Carathéodory length of $\gamma(s)$ is

$$
\ell(\gamma)=\int_{0}^{1}\left[\dot{x}^{2}(s)+\frac{\dot{y}^{2}(s)}{x^{2 m}(s)}\right]^{1 / 2} d s=\int_{0}^{1} \sqrt{\eta^{2} A^{2 m}} d s=\eta A^{m}=\sqrt{2 E}
$$

After detailed calculation, one has the following theorem. See Calin and Chang [6], Calin et al. [10], Chang et al. [13] and Chang and Li [15].

TheOREM 2. For any two points $P\left(x_{0}, y_{0}\right)$ and $Q\left(x_{1}, y_{0}\right)$ on the same horizontal line $y=y_{0}$, there is a unique geodesic connecting them.

If $y_{1}>0$, there are infinitely many geodesics $\gamma_{1}, \gamma_{2}, \ldots, \gamma_{k}, \ldots$ connecting $P(0,0)$ and $Q\left(0, y_{1}\right)$ with lengths $\ell_{1}, \ell_{2}, \ldots, \ell_{k}, \ldots$, where

$$
\ell_{k}=\left[a_{m}^{m}(m+1) k^{m} y_{1}\right]^{\frac{1}{m+1}} .
$$

For each $k \in \mathbb{N}$, there are two geodesics. Here $a_{m}=2 \int_{0}^{1} \frac{d t}{\sqrt{1-t^{2 m}}}$.

If $x_{1} \neq 0$, there is only a finite number of geodesics joining $P\left(x_{0}, y_{0}\right)$ and $Q\left(x_{1}, y_{1}\right)$. The number is approximated by

$$
N \approx\left[\frac{m+1}{a_{m}} \frac{2\left|y_{1}-y_{0}\right|}{x_{1}^{m+1}}\right] .
$$

3. Heat kernels for Grushin operators. In this section, we will construct the heat kernel for step $m+1$ Grushin operator $\Delta_{m}=\frac{1}{2} \frac{\partial^{2}}{\partial x^{2}}+\frac{1}{2} x^{2 m} \frac{\partial^{2}}{\partial y^{2}}$ and study the small time asymptotics of the heat kernel. Since the operator $\Delta_{m}$ is invariant under translations along the $y$-axis, we may assume that $y(0)=y_{0}=0$.

3.1. The modified action function. First we need the classical action integral $S(t)$. Recall that the associated Hamiltonian of Grushin operator is $H(x, y, \xi, \eta)=$ $\frac{1}{2}\left(\xi^{2}+x^{2 m} \eta^{2}\right)$ where $\xi$ and $\eta$ are dual variables of $x$ and $y$, respectively.

Along the bicharacteristic curves, we have

$$
\dot{x}=\xi, \quad \dot{y}=\eta x^{2 m} .
$$


The action integral is

$$
S(t)=\int_{0}^{t}(\xi \cdot \dot{x}+\eta \cdot \dot{y}-H) d s=\int_{0}^{t} H d s .
$$

$H$ is a constant along a bicharacteristic curve. From the expression of $H$ and (3.1), one has

$$
H=\frac{1}{2}\left(\dot{x}^{2}+\eta^{2} x^{2 m}\right)=\frac{1}{2} \eta^{2} A^{2 m},
$$

where $A$ is a parameter representing the amplitude of $x(s)$. Coupling the last two equations yields

$$
S(t)=\frac{1}{2} \eta^{2} A^{2 m} t
$$

Recall that, along the bicharacteristic curves one has

$$
\begin{gathered}
y(t)-y(0)=\eta \int_{0}^{t} x^{2 m}(s) d s, \\
x(s)=A \mathcal{S}_{m}\left(p\left(s+s_{0}\right)\right), \quad 0 \leq s_{0} \leq a_{m},
\end{gathered}
$$

and

$$
x_{0}=x(0)=A \mathcal{S}_{m}\left(p s_{0}\right), \quad x=x(t)=A \mathcal{S}_{m}\left(p\left(t+s_{0}\right)\right)
$$

where $\mathcal{S}_{m}(s):=x(s ; 1,1)$ and $x(s ; A, p)=A x(p s ; 1,1)=A \mathcal{S}_{m}(p s)$ with $s \in \mathbb{R}$ and $p, A>0$. Moreover,

$$
p=\eta A^{m-1}
$$

We are going to find the integral in (3.4). Solving the Hamilton's system, one has

$$
\begin{aligned}
\int_{0}^{t} x^{2 m}(s) d s & =\frac{-1}{m \eta^{2}} \int_{0}^{t} x(s) \ddot{x}(s) d s \\
& =-\left.\frac{1}{k \eta^{2}} x(s) \dot{x}(s)\right|_{s=0} ^{s=t}+\frac{1}{m \eta^{2}} \int_{0}^{t}\left(\eta^{2} A^{2 m}-\eta^{2} x^{2 m}(s)\right) d s
\end{aligned}
$$

combining this equation with (3.5) and (3.6), one get

$$
\begin{aligned}
\eta^{2} \int_{0}^{t} x^{2 m}(s) d s & =\frac{1}{m+1}\left[-\left.x(s) \frac{d x(s)}{d s}\right|_{s=0} ^{s=t}+\eta^{2} A^{2 m} t\right] \\
& =\frac{1}{m+1}\left[-p x^{2} \frac{\mathcal{S}_{m}^{\prime}\left(p\left(t+s_{0}\right)\right)}{\mathcal{S}_{m}\left(p\left(t+s_{0}\right)\right)}+p x_{0}^{2} \frac{\mathcal{S}_{m}^{\prime}\left(p s_{0}\right)}{\mathcal{S}_{m}\left(p s_{0}\right)}+\eta^{2} A^{2 m} t\right] .
\end{aligned}
$$

From (3.3), (3.4) and (3.8), one has

$$
S(t)=\frac{1}{2} \eta^{2} A^{2 m} t=\frac{1}{2}\left[(m+1) \eta(y-y(0))+p x^{2} \frac{\mathcal{S}_{m}^{\prime}\left(p\left(t+s_{0}\right)\right)}{\mathcal{S}_{m}\left(p\left(t+s_{0}\right)\right)}-p x_{0}^{2} \frac{\mathcal{S}_{m}^{\prime}\left(p s_{0}\right)}{\mathcal{S}_{m}\left(p s_{0}\right)}\right] .
$$


As we know that the solution of the Hamilton-Jacobi equation $\frac{\partial h}{\partial t}+H\left(x, y, \frac{\partial h}{\partial x}, \frac{\partial h}{\partial y}\right)=0$ is

$$
\begin{aligned}
h(t) & =\eta y(0)+S(t) \\
& =\eta y+\frac{1}{2}\left[(m-1) \eta(y-y(0))+p x^{2} \frac{\mathcal{S}_{m}^{\prime}\left(p\left(t+s_{0}\right)\right)}{\mathcal{S}_{m}\left(p\left(t+s_{0}\right)\right)}-p x_{0}^{2} \frac{\mathcal{S}_{m}^{\prime}\left(p s_{0}\right)}{\mathcal{S}_{m}\left(p s_{0}\right)}\right] .
\end{aligned}
$$

To eliminate the term $y(0)$, we use (3.9) to obtain

$$
\eta(y-y(0))=\frac{1}{m+1}\left[\eta^{2} A^{2 m} t-p x^{2} \frac{\mathcal{S}_{m}^{\prime}\left(p\left(t+s_{0}\right)\right)}{\mathcal{S}_{m}\left(p\left(t+s_{0}\right)\right)}+p x_{0}^{2} \frac{\mathcal{S}_{m}^{\prime}\left(p s_{0}\right)}{\mathcal{S}_{m}\left(p s_{0}\right)}\right] .
$$

The last two equations yield

$$
\begin{aligned}
h(t) & =\eta y+\frac{1}{2(m+1)}\left[(m-1) \eta^{2} A^{2 m} t+2 p x^{2} \frac{\mathcal{S}_{m}^{\prime}\left(p\left(t+s_{0}\right)\right)}{\mathcal{S}_{m}\left(p\left(t+s_{0}\right)\right)}-2 p x_{0}^{2} \frac{\mathcal{S}_{m}^{\prime}\left(p s_{0}\right)}{\mathcal{S}_{m}\left(p s_{0}\right)}\right] \\
& =\frac{p y}{A^{m-1}}+\frac{1}{2(m+1)}\left[(m-1) p^{2} A^{2} t+2 p x^{2} \frac{\mathcal{S}_{m}^{\prime}\left(p\left(t+s_{0}\right)\right)}{\mathcal{S}_{m}\left(p\left(t+s_{0}\right)\right)}-2 p x_{0}^{2} \frac{\mathcal{S}_{m}^{\prime}\left(p s_{0}\right)}{\mathcal{S}_{m}\left(p s_{0}\right)}\right] .
\end{aligned}
$$

Here we have made use of (3.7) to get rid of $\eta$.

The representation of $h$ in (3.11) is not in the final form, since it involves $s_{0}$ and $A$, which can be eliminated by a investigation of (3.6) and the addition formula of $\mathcal{S}_{k}$. Moreover, $h\left(x, x_{0}, y, p, t\right)=\frac{1}{t} h\left(x, y, x_{0}, p t, 1\right)$. Set $f\left(x, y, x_{0}, \lambda\right):=h\left(x, y, x_{0}, \lambda, 1\right)$ with $\lambda=p t$. Now we are looking for a heat kernel in the form of $\frac{1}{t^{\alpha}} e^{-h}=\frac{1}{t^{\alpha}} e^{-\frac{f}{t}}$. However, the heat kernel should not depend on $\lambda$. To this end, we use a trick to get rid of $\lambda$ by summing over it. Thus we shall look for the heat kernel in the following form

$$
\mathcal{P}_{t}=\frac{1}{(2 \pi t)^{\alpha}} \int_{\Gamma} e^{-\frac{f\left(x, y, x_{0}, \lambda\right)}{t}} V(\lambda) d \lambda .
$$

Here $V(\lambda)$ is introduced for a good measure, which is called the volume element. The integral path may be deformed to a contour $\Gamma$ in the complex plane, such that the integration is taken on the best behavior of the integrand such that the integral is well-defined, for example, $\Gamma$ is the whole imaginary axis or the contour discussed in Chang et al. [14]. See also Beals et al. [2,3] and Calin et al. [8]. The function $f$ is called the modified (complex) action which plays the same role as the square of the distance function and satisfies the eiconal equation:

$$
\lambda \frac{\partial f}{\partial \lambda}+H\left(x, y, \frac{\partial f}{\partial x}, \frac{\partial f}{\partial y}\right)=f
$$

Furthermore, at the critical points $\lambda=\lambda_{j}$, i.e., $\frac{\partial f}{\partial \lambda}\left(\lambda_{j}\right)=0$, one has

$$
\begin{aligned}
f\left(x, y, 0, \lambda_{j}\right) & =\lambda_{j}\left[\frac{y}{x^{m-1}}\left|\mathcal{S}_{m}\left(\lambda_{j}\right)\right|^{m-1}+\frac{x^{2}}{2(m+1)} \frac{(m-1) \lambda_{j}+2 \mathcal{S}_{m}\left(\lambda_{j}\right) \mathcal{S}_{m}^{\prime}\left(\lambda_{j}\right)}{\mathcal{S}_{m}^{2}\left(\lambda_{j}\right)}\right] \\
& =\frac{\lambda_{j} x^{2}}{\mathcal{S}_{m}^{2}\left(\lambda_{j}\right)}\left[\mu\left(\lambda_{j}\right)+\frac{(m-1) \lambda+2 \mathcal{S}_{m}\left(\lambda_{j}\right) \mathcal{S}_{m}^{\prime}\left(\lambda_{j}\right)}{2(m+1)}\right] \\
& =\frac{\lambda_{j}^{2} x^{2}}{2 \mathcal{S}_{m}^{2}\left(\lambda_{j}\right)}=\frac{\ell_{j}^{2}(x, y)}{2},
\end{aligned}
$$


where $\ell_{j}(x, y)$ is the Carnot-Carathéodory length of the $j$-th geodesic connecting $(x, y)$ and the origin.

Hence, at the smallest critical point $\lambda_{1}, f$ takes its smallest value (on the real line). We have the following result and will discuss the detail in a forthcoming paper.

THEOREM 3. The heat kernel of step $(m+1)$ Grushin operator from the origin has the asymptotics

$$
\begin{aligned}
& \mathcal{P}_{t}(x, y ; 0,0) \\
\sim & \frac{1}{t^{\alpha}} e^{-\frac{d_{c c}^{2}(x, y)}{2 t}}\left[\frac{V\left(x, y, \lambda_{1}\right)}{x} \sqrt{\frac{2 \pi t}{\left|\left(\mathcal{S}_{m}\left(\lambda_{1}\right)+(m-1) \lambda_{1} \mathcal{S}_{m}^{\prime}\left(\lambda_{1}\right)\right) \mathcal{S}_{m}\left(\lambda_{1}\right) \mu^{\prime}\left(\lambda_{1}\right)\right|}}+\mathcal{O}(t)\right]
\end{aligned}
$$

as $t \rightarrow 0^{+}$. Here

$$
\mu(\lambda)=\frac{1}{m+1} \frac{\lambda-\mathcal{S}_{m}(\lambda) \mathcal{S}_{m}^{\prime}(\lambda)}{\left|\mathcal{S}_{m}(\lambda)\right|^{m+1}}
$$

and $\lambda_{1}$ is the smallest critical point of $f(x, y, 0, \lambda)$. As usual, $d_{c c}(x, y)$ is the CarnotCarathéodory distance between $(x, y)$ and the origin.

REMARK 4 . For the case of $m=1, \mathcal{S}_{1}(\cdot)=\sin (\cdot)$. Using the addition formula of the trigonometric functions, we have

$$
\begin{aligned}
& x^{2} \frac{\mathcal{S}_{1}^{\prime}\left(p\left(t+s_{0}\right)\right)}{\mathcal{S}_{1}\left(p\left(t+s_{0}\right)\right)}-x_{0}^{2} \frac{\mathcal{S}_{1}^{\prime}\left(p s_{0}\right)}{\mathcal{S}_{1}\left(p s_{0}\right)}=x^{2} \frac{\cos \left(p\left(t+s_{0}\right)\right)}{\sin \left(p\left(t+s_{0}\right)\right)}-x_{0}^{2} \frac{\cos \left(p s_{0}\right)}{\sin \left(p s_{0}\right)} \\
= & \left(x^{2}+x_{0}^{2}\right) \frac{\cos (p t)}{\sin (p t)}-x^{2} \frac{\sin \left(p s_{0}\right)}{\sin \left(p t+p s_{0}\right) \sin (p t)}-x_{0}^{2} \frac{\sin \left(p t+p s_{0}\right)}{\sin \left(p s_{0}\right) \sin (p t)} .
\end{aligned}
$$

Equations in (3.6) yield

$$
\frac{x}{x_{0}}=\frac{\sin \left(p t+p s_{0}\right)}{\sin \left(p s_{0}\right)}
$$

Combining the last two equations, one has

$$
x^{2} \frac{\mathcal{S}_{1}^{\prime}\left(p\left(t+s_{0}\right)\right)}{\mathcal{S}_{1}\left(p\left(t+s_{0}\right)\right)}-x_{0}^{2} \frac{\mathcal{S}_{1}^{\prime}\left(p s_{0}\right)}{\mathcal{S}_{1}\left(p s_{0}\right)}=\left(x^{2}+x_{0}^{2}\right) \frac{\cos (p t)}{\sin (p t)}-\frac{2 x x_{0}}{\sin (p t)} .
$$

Hence,

$$
\begin{aligned}
h(t) & =p y+p\left(\left(x^{2}+x_{0}^{2}\right) \frac{\cos (p t)}{\sin (p t)}-\frac{2 x x_{0}}{\sin (p t)}\right) \\
& =\eta y+\frac{\eta}{4}\left(\left(x-x_{0}\right)^{2} \cot \frac{\eta t}{2}-\left(x+x_{0}\right)^{2} \tan \frac{\eta t}{2}\right),
\end{aligned}
$$

since $p=\eta A^{1-1}=\eta$. We recover the result in Calin et al. [10] and Chang et al. [13]. Our approach here to find $h(t)$ is easier and more convenient to generalize to higher step cases.

REMARK 5. For the case of $m=2$, the function $\mathcal{S}_{2}$ is the Jacobi elliptic function with modulus $i$ : $\mathcal{S}_{2}(\cdot)=\operatorname{sn}(\cdot ; i):=\operatorname{sn}(\cdot)$. The following result is well-known (see Lawden [27]):

$$
\operatorname{sn}^{\prime}(u)=\operatorname{cn}(u) \operatorname{dn}(u),
$$


and the addition formula

$$
\operatorname{sn}(u+v)=\frac{\operatorname{sn}(u) \operatorname{sn}^{\prime}(v)+\operatorname{sn}(v) \operatorname{sn}^{\prime}(u)}{1+\operatorname{sn}^{2}(u) \operatorname{sn}^{2}(v)} .
$$

From (3.17) and (3.18), one has

$$
\begin{aligned}
& x^{2} \frac{\mathcal{S}_{2}^{\prime}\left(p\left(t+s_{0}\right)\right)}{\mathcal{S}_{2}\left(p\left(t+s_{0}\right)\right)}-x_{0}^{2} \frac{\mathcal{S}_{2}^{\prime}\left(p s_{0}\right)}{\mathcal{S}_{2}\left(p s_{0}\right)}=x^{2} \frac{\mathrm{sn}^{\prime}\left(p t+p s_{0}\right)}{\operatorname{sn}\left(p t+p s_{0}\right)}-x_{0}^{2} \frac{\mathrm{sn}^{\prime}\left(p s_{0}\right)}{\operatorname{sn}\left(p s_{0}\right)} \\
= & \left(x^{2}+x_{0}^{2}\right) \frac{\operatorname{sn}^{\prime}(p t)}{\operatorname{sn}(p t)}-x^{2} \frac{\operatorname{sn}\left(p s_{0}\right)\left[1+\operatorname{sn}^{2}\left(p t+p s_{0}\right) \operatorname{sn}^{2}(p t)\right]}{\operatorname{sn}\left(p t+p s_{0}\right) \operatorname{sn}(p t)} \\
& -x_{0}^{2} \frac{\operatorname{sn}\left(p t+p s_{0}\right)\left[1+\operatorname{sn}^{2}\left(p s_{0}\right) s n^{2}(p t)\right]}{\operatorname{sn}\left(p s_{0}\right) \operatorname{sn}(p t)} .
\end{aligned}
$$

Equation (3.6) yields

$$
x=A \operatorname{sn}\left(p t+p s_{0}\right), \quad x_{0}=A \operatorname{sn}\left(p s_{0}\right) .
$$

Hence,

$$
\begin{aligned}
& x^{2} \frac{\mathcal{S}_{2}^{\prime}\left(p\left(t+s_{0}\right)\right)}{\mathcal{S}_{2}\left(p\left(t+s_{0}\right)\right)}-x_{0}^{2} \frac{\mathcal{S}_{2}^{\prime}\left(p s_{0}\right)}{\mathcal{S}_{2}\left(p s_{0}\right)} \\
= & \left(x^{2}+x_{0}^{2}\right) \frac{\operatorname{sn}^{\prime}(p t)}{\operatorname{sn}(p t)}-\frac{x x_{0}}{\operatorname{sn}(p t)}\left[2+\operatorname{sn}^{2}(p t) \frac{x^{2}+x_{0}^{2}}{A^{2}}\right] .
\end{aligned}
$$

If $x x_{0} \neq 0$, we need find $A$. In view of (3.6), we just need find $\operatorname{sn}\left(p s_{0}\right)$. By the addition formula

$$
\operatorname{sn}\left(p t+p s_{0}\right)=\frac{\operatorname{sn}\left(p s_{0}\right) \operatorname{sn}^{\prime}(p t)+\operatorname{sn}(p t) \operatorname{sn}^{\prime}\left(p s_{0}\right)}{1+\operatorname{sn}^{2}\left(p s_{0}\right) \operatorname{sn}^{2}(p t)}
$$

one has

$$
\frac{x}{x_{0}}=\frac{\operatorname{sn}\left(p t+p s_{0}\right)}{\operatorname{sn}\left(p s_{0}\right)}=\frac{\operatorname{sn}\left(p s_{0}\right) \operatorname{sn}^{\prime}(p t) \pm \operatorname{sn}(p t) \sqrt{1-\mathrm{sn}^{4}\left(p s_{0}\right)}}{\operatorname{sn}\left(p s_{0}\right)\left[1+\mathrm{sn}^{2}\left(p s_{0}\right) \mathrm{sn}^{2}(p t)\right]} .
$$

Therefore, $T=\operatorname{sn}\left(p s_{0}\right)$ satisfies the following equation

$$
B^{2} \mathrm{~s}^{4} T^{6}+\left(2 B^{2}-2 B \mathrm{~s}^{\prime}+1\right) \mathrm{s}^{2} T^{4}+\left(B-\mathrm{s}^{\prime}\right)^{2} T^{2}=\mathrm{s}^{2},
$$

where $\mathrm{s}=\mathrm{sn}(p t), \mathrm{s}^{\prime}=\mathrm{sn}^{\prime}(p t)$ and $B=x / x_{0}$. This equation can be solved in an explicit although complicated form by regarding it as a cubic equation of $T^{2}$. It follows that $T=\operatorname{sn}\left(p s_{0}\right)$ is a function of $x, x_{0}$ and $p t$, so is

$$
A=x_{0} / \operatorname{sn}\left(p s_{0}\right):=A\left(x, x_{0}, p t\right)
$$

Now (3.11) leads to

$$
\begin{aligned}
h(t) & =\eta y(0)+S(t) \\
& =p \frac{y}{A}+\frac{p}{6}\left[p A^{2} t+2\left(x^{2}+x_{0}^{2}\right) \frac{\operatorname{sn}^{\prime}(p t)}{\operatorname{sn}(p t)}-2 \frac{x x_{0}}{\operatorname{sn}(p t)}\left(2+\operatorname{sn}^{2}(p t) \frac{x^{2}+x_{0}^{2}}{A^{2}}\right)\right] .
\end{aligned}
$$


When $x_{0}=0$ and $x>0$, from (3.6) one has $s_{0}=0$ and $A=\frac{x}{|\operatorname{sn}(p t)|}$, which lead to

$$
\begin{aligned}
h(t) & =\frac{p}{6}\left[\frac{6 y}{x}|\operatorname{sn}(p t)|+x^{2} \frac{p t}{\operatorname{sn}^{2}(p t)}+2 x^{2} \frac{\operatorname{sn}^{\prime}(p t)}{\operatorname{sn}(p t)}\right] \\
& =p\left[\frac{y}{x}|\operatorname{sn}(p t)|+x^{2} \frac{p t+2 \operatorname{sn}(p t) \operatorname{sn}^{\prime}(p t)}{6 \operatorname{sn}^{2}(p t)}\right] .
\end{aligned}
$$

Thus we can define the modified action function

$$
f\left(x, y, x_{0}, \lambda\right)=\lambda \frac{y}{A}+\frac{\lambda}{6}\left[\lambda A^{2}+2\left(x^{2}+x_{0}^{2}\right) \frac{\operatorname{sn}^{\prime} \lambda}{\operatorname{sn} \lambda}-2 \frac{x x_{0}}{\operatorname{sn} \lambda}\left(2+\operatorname{sn}^{2} \lambda \frac{x^{2}+x_{0}^{2}}{A^{2}}\right)\right],
$$

where $A=A\left(x, x_{0}, \lambda\right)$ is a function depending only on $x, x_{0}$ and $\lambda$. Moreover, if $x_{0}=0$ and $x>0$, one has $x=A|\operatorname{sn} \lambda|$. In this case, the modified action function reduces to

$$
\begin{aligned}
f\left(x, y, x_{0}, \lambda\right) & =\lambda \frac{y}{A}+\frac{1}{6}\left[\lambda^{2} A^{2}+2 x^{2} \frac{\mathrm{sn}^{\prime} \lambda}{\operatorname{sn} \lambda}\right] \\
& =\lambda\left[\frac{y}{x}|\operatorname{sn} \lambda|+x^{2} \frac{\lambda+2 \operatorname{sn} \lambda \operatorname{sn}^{\prime} \lambda}{6 \operatorname{sn}^{2} \lambda}\right] .
\end{aligned}
$$

3.2. The volume element. In this section, we will show that for higher $(>2)$ step cases, the volume element can be found in a similar way as the step 2 case. The argument here is essentially similar as in Calin et al. [8] and Greiner [22]. Assume generally that

$$
\Delta_{\mathbf{X}}=\frac{1}{2} \sum_{k=1}^{\ell} X_{k}^{2}
$$

By the definition of the heat kernel, one needs to show

$$
\begin{cases}\mathcal{P}_{t}\left(x, x_{0}, y\right)>0, & t>0 \text { and }(x, y) \in \mathbb{R}^{2 n} \\ \left(\Delta_{\mathbf{X}}-\frac{\partial}{\partial t}\right) \mathcal{P}_{t}=0, & t>0 \\ \lim _{t \rightarrow 0} \mathcal{P}_{t}\left(x, x_{0}, y\right)=\delta\left(x-x_{0}\right) \delta(y) . & \end{cases}
$$

The positivity of the heat kernel was proved in Gaveau [18] and Hulanicki [26] by probabilistic method since the heat kernel is the density function of a stochastic process. Recently, we had present an analytic approach to this property in Chang et al. [14]. Now we turn to the second condition in (3.24). In view of (3.12), we need to look at the term $\left(\Delta_{\mathbf{X}}-\frac{\partial}{\partial t}\right) \frac{e^{-f / t} V}{t^{\alpha}}$. Since $\Delta_{\mathbf{X}} f$ is a function depending on the variables $x$ and $y$, the volume element $V=V(x, y, \lambda)$ depends on the spatial variables in general. Therefore,

$$
\begin{aligned}
\Delta_{\mathbf{X}}\left(e^{-f / t} V\right) & =\Delta_{\mathbf{X}}\left(e^{-f / t}\right) V+\sum_{k=1}^{\ell} X_{k}\left(e^{-f / t}\right) X_{k} V+e^{-f / t} \Delta_{\mathbf{X}} V \\
& =e^{-f / t}\left[\left(\frac{H(\nabla f)}{t^{2}}-\frac{\Delta_{\mathbf{X}} f}{t}\right) V-\frac{1}{t} X f \cdot X V+\Delta_{\mathbf{X}} V\right]
\end{aligned}
$$


and

$$
\frac{\partial}{\partial t} \frac{e^{-f / t} V}{t^{\alpha}}=\frac{e^{-f / t} V}{t^{\alpha}}\left(\frac{f}{t^{2}}-\frac{\alpha}{t}\right)
$$

Hence,

$$
\left(\Delta_{\mathbf{x}}-\frac{\partial}{\partial t}\right) \frac{e^{-f / t} V}{t^{\alpha}}=\frac{e^{-f / t}}{t^{\alpha}}\left[\frac{(H(\nabla f)-f) V}{t^{2}}-\frac{\left(\Delta_{\mathbf{x}} f-\alpha\right) V+X f \cdot X V}{t}+\Delta_{\mathbf{x}} V\right] .
$$

Now the eiconal equation $\lambda \frac{\partial f}{\partial \lambda}+H(\nabla f)=f$ implies that

$$
\begin{aligned}
e^{-f / t} \frac{(H(\nabla f)-f) V}{t^{2}} & =\frac{1}{t} e^{-f / t}\left(-\frac{1}{t} \lambda \frac{\partial f}{\partial \lambda}\right) \lambda V \\
& =\frac{1}{t}\left(\frac{\partial}{\partial \lambda}\left(e^{-f / t} \lambda V\right)-e^{-f / t} \frac{\partial}{\partial \lambda}(\lambda V)\right),
\end{aligned}
$$

which leads to

$(3.25)$

$$
\begin{aligned}
& \left(\Delta_{\mathbf{X}}-\frac{\partial}{\partial t}\right) \frac{e^{-f / t} V}{t^{\alpha}} \\
= & -\frac{e^{-f / t}}{t^{\alpha+1}}\left[\lambda \frac{\partial V}{\partial \lambda}+X f \cdot X V+\left(\Delta_{\mathbf{X}} f-\alpha+1\right) V\right]+\frac{e^{-f / t}}{t^{\alpha}} \Delta_{\mathbf{X}} V+\frac{\partial}{\partial \lambda} \frac{e^{-f / t} \lambda V}{t^{\alpha+1}} .
\end{aligned}
$$

The next step is to make the first term on the right-hand side be the power $(-\alpha)$ of $t$. To this end, we set

$$
V=-f_{\lambda} v
$$

Differentiating the eiconal equation with respect to $\lambda$, we get

$$
\lambda f_{\lambda \lambda}+X f \cdot X f_{\lambda}=0 .
$$

Then the first term on the right-hand side of (3.25) becomes

$$
\begin{aligned}
& -\frac{e^{-f / t}}{t^{\alpha+1}}\left[\lambda \frac{\partial V}{\partial \lambda}+X f \cdot X V+\left(\Delta_{\mathbf{X}} f-\alpha+1\right) V\right] \\
= & \frac{e^{-f / t}}{t^{\alpha+1}}\left[\lambda \frac{\partial}{\partial \lambda}\left(f_{\lambda} v\right)+X f \cdot X\left(f_{\lambda} v\right)+\left(\Delta_{\mathbf{X}} f-\alpha+1\right) f_{\lambda} v\right] \\
= & -\frac{\partial}{\partial \lambda}\left(\frac{e^{-f / t}}{t^{\alpha}}\left[\lambda v_{\lambda}+X f \cdot X v+\left(\Delta_{\mathbf{X}} f-\alpha+1\right) v\right]\right) \\
& +\frac{e^{-f / t}}{t^{\alpha}} \frac{\partial}{\partial \lambda}\left[\lambda v_{\lambda}+X f \cdot X v+\left(\Delta_{\mathbf{X}} f-\alpha+1\right) v\right] .
\end{aligned}
$$

Moreover,

$$
\Delta_{\mathbf{X}} V=\Delta_{\mathbf{X}}\left(-f_{\lambda} v\right)=-v \Delta_{\mathbf{X}} f_{\lambda}-X f_{\lambda} X v-f_{\lambda} \Delta_{\mathbf{X}} v
$$

Thus

$$
\begin{aligned}
& \frac{\partial}{\partial \lambda}\left[\lambda v_{\lambda}+X f \cdot X v+\left(\Delta_{\mathbf{X}} f-\alpha+1\right) v\right]+\Delta_{\mathbf{X}}\left(-f_{\lambda} v\right) \\
= & \lambda v_{\lambda \lambda}+X f \cdot X v_{\lambda}+\left(\Delta_{\mathbf{X}} f-\alpha+2\right) v_{\lambda}-f_{\lambda} \Delta_{\mathbf{X}} v \\
= & \left(T+\left(\Delta_{\mathbf{X}} f-\alpha+2\right)\right) \frac{\partial v}{\partial \lambda}-f_{\lambda} \Delta_{\mathbf{X}} v
\end{aligned}
$$


where

$$
T=\lambda \frac{\partial}{\partial \lambda}+X f \cdot X
$$

Substituting the last two equations into

$$
\frac{\partial S}{\partial y}\left(t, x, y, x_{0}, \eta\right)=\eta(t)-\eta(0) \frac{\partial y\left(0 ; x, y, x_{0}, \eta, t\right)}{\partial y}
$$

leads to

$$
\begin{aligned}
\left(\Delta_{\mathbf{X}}-\frac{\partial}{\partial t}\right) \frac{e^{-f / t} V}{t^{\alpha}}= & \frac{e^{-f / t}}{t^{\alpha}}\left(\left(T+\left(\Delta_{\mathbf{X}} f-\alpha+2\right)\right) \frac{\partial v}{\partial \lambda}-f_{\lambda} \Delta_{\mathbf{X}} v\right) \\
& -\frac{\partial}{\partial \lambda} \frac{e^{-f / t} \lambda f_{\lambda} v}{t^{\alpha+1}}-\frac{\partial}{\partial \lambda}\left(\frac{e^{-f / t}}{t^{\alpha}}\left(T v+\left(\Delta_{\mathbf{X}} f-\alpha+1\right) v\right)\right) .
\end{aligned}
$$

We have shown that

TheOREM 6. The kernel (3.12) is a solution of

$$
\frac{\partial \mathcal{P}_{t}}{\partial t}-\Delta_{\mathbf{X}} \mathcal{P}_{t}=0, \quad t>0
$$

if $v$ is a solution of

$$
\left(T+\left(\Delta_{\mathbf{X}} f-\alpha+2\right)\right) \frac{\partial v}{\partial \lambda}-f_{\lambda} \Delta_{\mathbf{X}} v=0
$$

and

$$
\frac{e^{-f / t} \lambda f_{\lambda} v}{t^{\alpha+1}} \quad \text { and } \quad \frac{e^{-f / t}}{t^{\alpha}}\left(T v+\left(\Delta_{\mathbf{X}} f-\alpha+1\right) v\right)
$$

both vanish at the end points of the contour $\Gamma$.

The equation (3.29) is called the generalized transport equation. If the volume element $V$ does not depend on the spatial variables $x$ and $y$, then (3.25) simplifies to

$$
\left(\Delta_{\mathbf{X}}-\frac{\partial}{\partial t}\right) \frac{e^{-f / t} V}{t^{\alpha}}=-\frac{e^{-f / t}}{t^{\alpha+1}}\left[\lambda \frac{\partial V}{\partial \lambda}+\left(\Delta_{\mathbf{X}} f-\alpha+1\right) V\right]+\frac{\partial}{\partial \lambda} \frac{e^{-f / t} \lambda V}{t^{\alpha+1}}
$$

Then Theorem 6 can be written into a more elegant form:

THEOREM 7. The kernel (3.12) is a solution of (3.28) if $V(\lambda)$ does not depend on $x, y$ and is a solution of

$$
\lambda \frac{\partial V}{\partial \lambda}+\left(\Delta_{\mathbf{x}} f-\alpha+1\right) V=0
$$

and $\frac{e^{-f / t} \lambda V}{t^{\alpha+1}}$ vanishes at the end points (might be infinity) of the contour $\Gamma$.

Note that (3.32) is a first order transport equation. This is the case of step 2, because of the fact that $\Delta f$ does not depend on the spatial variables in this case. Examples are heat kernels of Heisenberg subLaplacian and step 2 Grushin operator; see Beals et al. [4], Calin and Chang [6], Greiner [22]. For higher $(>2)$ step cases, $\Delta f$ depends on the spatial variables, in which we need solve for $V$ like in Theorem 6 . 
However Theorem 6 is not so convenient. Since we are going to find the small time asymtotics of the heat kernel by the steepest descent method, in which the main contribution comes from the saddle point of the integral, i.e., at the points where $f_{\lambda}$ vanishes. But the integrand also vanishes in (3.12) at the saddle points. We will try an alternative way to find the volume element $V(\lambda)$.

Assume $V=W_{\lambda}$. Write the second term on the right-hand side of (3.25) as

$$
\frac{e^{-f / t}}{t^{\alpha}} \Delta W_{\lambda}=\frac{\partial}{\partial \lambda}\left(\frac{e^{-f / t}}{t^{\alpha}} \Delta W\right)+\frac{e^{-f / t} f_{\lambda}}{t^{\alpha+1}} \Delta W .
$$

Hence we have the following result:

THEOREM 8. The kernel (3.12) is a solution of (3.28) if $W$ is a solution of

$$
\lambda \frac{\partial^{2} W}{\partial \lambda^{2}}+(\Delta f-\alpha+1) \frac{\partial W}{\partial \lambda}+f_{\lambda} \Delta W=0
$$

and

$$
\frac{e^{-f / t} \lambda f_{\lambda} W}{t^{\alpha+1}} \quad \text { and } \quad \frac{e^{-f / t}}{t^{\alpha}} \Delta W
$$

both vanish at the end points (might be infinity) of the contour $\Gamma$.

3.2.1. The case for $m=1$. The volume element could be computed as in Calin et al. [8] and Chang et al. [13]. We start with the second assertion of the above system. Note that

$$
\left(\Delta_{1}-\frac{\partial}{\partial t}\right) \frac{e^{-\frac{f}{t}} V(\tau)}{t^{\alpha}}=-\frac{e^{-\frac{f}{t}}}{t^{\alpha+1}}\left[\tau \frac{d V}{d \tau}+\left(\Delta_{1} f-\alpha+1\right) V\right]+\frac{\partial}{\partial \tau}\left(\tau \frac{e^{-\frac{f}{t}} V(\tau)}{t^{\alpha}}\right)
$$

Assuming

$$
\lim _{\tau \rightarrow \pm \infty} \tau e^{-\frac{f}{t}} V(\tau)=0
$$

one has

$$
\begin{aligned}
& \left(\Delta_{1}-\frac{\partial}{\partial t}\right) \frac{1}{t^{\alpha}} \int_{\mathbb{R}} e^{-\frac{f}{t}} V(\tau) d \tau \\
= & -\frac{1}{t^{\alpha+1}} \int_{\mathbb{R}} e^{-\frac{f}{t}}\left[\tau \frac{d V}{d \tau}+\left(\Delta_{1} f-\alpha+1\right) V(\tau)\right] d \tau=0, \quad \forall t>0 .
\end{aligned}
$$

Since $\Delta_{1} f=\frac{\tau}{2} \operatorname{coth} \tau$, then we have

$$
\tau \frac{\partial V}{\partial \tau}+\left(\frac{1}{2} \tau \operatorname{coth} \tau+1-\alpha\right) V=0
$$

which is so-called the transport equation. Hence,

$$
V(\tau)=\frac{(c \tau)^{\alpha-1}}{\sqrt{\sinh \tau}}
$$

We need $V$ holomorphic near $\tau=0$. This forces us to choose $\alpha=n+\frac{1}{2}, n=1,2, \ldots$ and the volume element is

$$
V(\tau)=c \tau^{n-1} \sqrt{\frac{\tau}{\sinh \tau}}
$$


Set $V(\tau)=\sum_{k=0}^{\infty} a_{k} \tau^{k}$. Substituting this into the transport equation, one has

$$
\begin{aligned}
& \sum_{k=1}^{\infty} a_{k} \tau^{k}+\left(\frac{1}{2} \tau \operatorname{coth} \tau-\alpha+1\right)\left(\sum_{k=0}^{\infty} a_{k} \tau^{k}\right) \\
= & a_{1} \tau+\frac{3}{2} a_{0}+\frac{3}{2} a_{1} \tau-\alpha a_{0}-\alpha a_{1} \tau+\cdots=0
\end{aligned}
$$

or

$$
\left(\frac{3}{2}-\alpha\right) a_{0}+\mathcal{O}(\tau)=0
$$

Since $V(0) \neq 0$, we have $n=1$ and $\alpha=\frac{3}{2}$. Therefore, the heat kernel of the step 2 Grushin operator is

$$
\begin{aligned}
\mathcal{P}_{t}\left(x, x_{0}, y\right) & =\frac{1}{(2 \pi t)^{\frac{3}{2}}} \int_{\mathbb{R}} e^{-\frac{f\left(x, x_{0}, y, \tau\right)}{t}} V(\tau) d \tau \\
& =\frac{1}{(2 \pi t)^{\frac{3}{2}}} \int_{\mathbb{R}} e^{\frac{\tau}{2 t}\left\{2 i y-\frac{1}{2 \sinh \tau}\left[\left(x+x_{0}\right)^{2} \tanh \frac{\tau}{2}+\left(x-x_{0}\right)^{2} \operatorname{coth} \frac{\tau}{2}\right]\right\}}\left(\frac{\tau}{\sinh \tau}\right)^{\frac{1}{2}} d \tau .
\end{aligned}
$$

3.2.2. The case for $m=2$. In this case, the volume element $V(x, y, \lambda)$ can be found in the following way. If we assume $\Delta_{2} V=0$, from (3.25), $V$ satisfies

$$
\lambda \frac{\partial V}{\partial \lambda}+X(f) X(V)+Y(f) Y(V)+\left(\Delta_{2} f+1-\alpha\right) V=0
$$

We further assume that the solution $V$ of (3.36) does not depend on $y$ and has the form of

$$
V(x, y, \lambda)=V_{1}(x) V_{2}(\lambda)
$$

Then equation (3.36) reduces to

$$
\lambda V_{1}(x) V_{2}^{\prime}(\lambda)+f_{x} V_{1}^{\prime}(x) V_{2}(\lambda)+\left(\frac{1}{2} f_{x x}+1-\alpha\right) V_{1}(x) V_{2}(\lambda)=0 .
$$

To eliminate the terms involving $y$ in $f_{x}$ and $f_{x x}$, we put $\frac{V_{1}^{\prime}(x)}{V_{1}(x)}=\frac{1}{x}$. Thus, we may set $V_{1}(x)=x$. Note that

$$
\Delta_{2} V=\Delta_{2}\left[V_{1}(x) V_{2}(\lambda)\right]=V_{2}(\lambda) \Delta_{2} x=0 .
$$

Coupling (3.23) and (3.38), $V_{2}$ satisfies the following equation

$$
V_{2}^{\prime}(\lambda)+\left(\frac{\lambda+2 \operatorname{sn} \lambda \mathrm{sn}^{\prime} \lambda}{2 \mathrm{sn}^{2} \lambda}+\frac{1-\alpha}{\lambda}\right) V_{2}(\lambda)=0 .
$$

Thus

$$
\log V_{2}(\lambda)=-\int\left(\frac{\lambda}{2 \operatorname{sn}^{2} \lambda}+\frac{\operatorname{sn}^{\prime} \lambda}{\operatorname{sn} \lambda}+\frac{1-\alpha}{\lambda}\right) d \lambda=\log \left|\frac{\lambda^{\alpha-1}}{\operatorname{sn} \lambda}\right|-\int \frac{\lambda}{2 \operatorname{sn}^{2} \lambda} d \lambda .
$$

To evaluate the integral in the last equation, we recall an integral formula of the sn function:

$$
F(u):=\int_{0}^{u} \frac{d v}{\operatorname{sn}^{2} v}=u-E(u)-\frac{\operatorname{sn}^{\prime} u}{\operatorname{sn} u}
$$


where $E(u)$ is the elliptic integral of the second kind

$$
E(u)=\int_{0}^{u} \mathrm{dn}^{2} v d v
$$

see Exercise 8.2.2(b) on p.211 and p.129 in [1]. Then integration by parts leads to

$$
\begin{aligned}
2 I(\lambda) & :=\int_{0}^{\lambda} \frac{u}{\operatorname{sn}^{2} u} d u=\lambda F(\lambda)-\int_{0}^{\lambda} F(u) d u \\
& =\frac{\lambda^{2}}{2}-\lambda E(\lambda)-\lambda \frac{\operatorname{sn}^{\prime} \lambda}{\operatorname{sn} \lambda}+\log |\operatorname{sn} \lambda|+\int_{0}^{\lambda} E(u) d u .
\end{aligned}
$$

Moreover,

$$
e^{-I(\lambda)} \sim e^{-\frac{1}{2}}|\operatorname{sn} \lambda|^{-\frac{1}{2}} \quad \text { as } \quad \lambda \rightarrow 0
$$

and there is a constant $M>0$, such that $\left|e^{-I(\lambda)}\right| \leq M e^{-\lambda^{2}}$. Hence, one has

$$
V_{2}(\lambda)=C\left|\frac{\lambda^{\alpha-1}}{\operatorname{sn} \lambda}\right| e^{-I(\lambda)},
$$

and

$$
V(x, y, \lambda)=V_{1}(x) V_{2}(\lambda)=C x\left|\frac{\lambda^{\alpha-1}}{\operatorname{sn} \lambda}\right| e^{-I(\lambda)} .
$$

Recall that

$$
\operatorname{sn} \lambda \sim \lambda \quad \text { as } \quad \lambda \rightarrow 0 .
$$

For $V(\lambda)$ is smooth near $\lambda=0$, we choose $\alpha=n+\frac{5}{2}, n=0,1, \cdots$, and

$$
V(x, y, \lambda)=C x \lambda^{n}\left|\frac{\lambda^{\frac{3}{2}}}{\operatorname{sn} \lambda}\right| e^{-I(\lambda)} .
$$

Thus we are looking for the heat kernel in the following form

$$
\mathcal{P}_{t}(x, y ; 0,0)=\frac{C}{(2 \pi t)^{n+\frac{5}{2}}} \int_{\mathbb{R}} e^{-\frac{f(x, y, 0, \lambda)}{t}} V(x, \lambda) d \lambda .
$$

If we choose $\alpha=\frac{5}{2}$ (i.e., $n=0$ ), then

$$
V_{2}(\lambda)=C \frac{|\lambda|^{\frac{3}{2}}}{|\sin \lambda|} e^{-I(\lambda)} .
$$

Denote $a_{2}=\frac{1}{4} \sqrt{\frac{2}{\pi}}\left(\Gamma\left(\frac{1}{4}\right)\right)^{2}$ and

$$
\begin{aligned}
I & :=\frac{1}{C} \int_{-a_{2}}^{a_{2}} V_{2}\left(\lambda_{1}\right) \sqrt{\frac{\mu^{\prime}\left(\lambda_{1}\right)}{\operatorname{sn} \lambda_{1}+\lambda_{1} \operatorname{sn}^{\prime} \lambda_{1}}}\left(\frac{\operatorname{sn} \lambda_{1}}{\lambda_{1}}\right)^{4} d \lambda_{1} \\
& =\int_{-a_{2}}^{a_{2}}|\lambda|\left(\frac{\lambda}{\operatorname{sn} \lambda}\right)^{\frac{1}{2}} e^{-I(\lambda)} \sqrt{\frac{\left|\operatorname{sn} \lambda-\lambda \operatorname{sn}^{\prime} \lambda\right|}{\operatorname{sn}^{4} \lambda\left|\operatorname{sn} \lambda+\lambda \operatorname{sn}^{\prime} \lambda\right|}}\left(\frac{\operatorname{sn} \lambda}{\lambda}\right)^{4} d \lambda \\
& =2 \int_{0}^{a_{2}} \sqrt{\frac{\left(\operatorname{sn} \lambda-\lambda \operatorname{sn}^{\prime} \lambda\right) \operatorname{sn}^{3} \lambda}{\left(\operatorname{sn} \lambda+\lambda \operatorname{sn}^{\prime} \lambda\right) \lambda^{5}}} e^{-I(\lambda)} d \lambda .
\end{aligned}
$$


If we choose the constant

$$
C=\frac{1}{4 \sqrt{2 \pi} I}
$$

Then

$$
\lim _{t \rightarrow 0^{+}} \int_{-\infty}^{\infty} \int_{-\infty}^{\infty} \mathcal{P}_{t}(x, y) d x d y=\lim _{t \rightarrow 0^{+}} \int_{-\infty}^{\infty} \int_{-\infty}^{\infty} \int_{-\infty}^{\infty} e^{-\frac{f(x, y, \lambda)}{t}} V(x, \lambda) d \lambda d x d y=1 .
$$

Thus we have shown

TheOREm 9. The heat kernel of the step 3 Grushin operator $(m=2)$ from the origin is

$$
\mathcal{P}_{t}(x, y ; 0,0)=\frac{1}{t^{\frac{5}{2}}} \int_{\Gamma} e^{-\frac{f(x, y, 0, \lambda)}{t}} x V_{2}(\lambda) d \lambda
$$

where $f$ and $V_{2}$ are given in (3.23) and (3.45), respectively.

The heat kernel from the point $\left(x_{0}, 0\right)$ with $x_{0} \neq 0$ is much more complicated. In this situation, one needs to solve the generalized transport equation (3.34) to find the volume element $V(x, y, \lambda)$. Here is the theorem.

THEOREM 10. The heat kernel of step 3 Grushin operator from the point $\left(x_{0}, 0\right)$ with $x_{0} \neq 0$ is

$$
\mathcal{P}_{t}\left(x, y ; x_{0}, 0\right)=\frac{1}{t^{\frac{5}{2}}} \int_{\mathbb{R}} e^{-\frac{f\left(x, y, x_{0}, \lambda\right)}{t}} V(x, y, \lambda) d \lambda,
$$

where $f\left(x, y, x_{0}, \lambda\right)$ is given in (3.22) and $V(x, y, \lambda)=W_{\lambda}(x, y, \lambda)$ with $W$ is a solution of the generalized transport equation (3.34).

3.2.3. Heat kernels: the general case $m>2$. In this case, the modified function $f\left(x, y, x_{0}, \lambda\right)$ can be obtained by knowledge about the addition formula of $\mathcal{S}_{m}(\cdot)$, the Abelian functions. In particular, the formula can be worked out nicely when $x_{0}=0$ as in (3.21) and (3.23).

Assume $x_{0}=0$ and $x>0$. One has $s_{0}=0$ and $x=A\left|\mathcal{S}_{m}(t)\right|$. From (3.11), we get

$$
\begin{aligned}
h(t) & =\frac{p}{A^{m-1}} y+\frac{1}{2(m+1)}\left[(m-1) p^{2} A^{2} t+2 p x^{2} \frac{\mathcal{S}_{m}^{\prime}(p t)}{\mathcal{S}_{m}(p t)}\right] \\
& =p\left[\frac{y}{x^{m-1}}\left|\mathcal{S}_{m}(p t)\right|^{m-1}+\frac{x^{2}}{2(m+1)} \frac{(m-1) p t+2 \mathcal{S}_{m}(p t) \mathcal{S}_{m}^{\prime}(p t)}{\mathcal{S}_{m}^{2}(p t)}\right] .
\end{aligned}
$$

And

$$
f(x, y, 0, \lambda)=\lambda\left[\frac{y}{x^{m-1}}\left|\mathcal{S}_{m}(\lambda)\right|^{m-1}+\frac{x^{2}}{2(m+1)} \frac{(m-1) \lambda+2 \mathcal{S}_{m}(\lambda) \mathcal{S}_{m}^{\prime}(\lambda)}{\mathcal{S}_{m}^{2}(\lambda)}\right]
$$

From this,

$$
f(x, y, 0, \lambda) \rightarrow+\infty \quad \text { as } \quad \lambda \rightarrow \pm \infty
$$


Hence $e^{-\frac{f(x, y, 0, \lambda)}{t}} V(x, y, \lambda) \rightarrow 0$ exponentially as $\lambda \rightarrow \pm \infty$ for $t>0$. The singularities of $f$ on the real line are $\lambda=j a_{m}, j= \pm 1, \pm 2, \cdots$, at which

$$
f(x, y, 0, \lambda) \rightarrow+\infty \text {. }
$$

Thus the integral in (3.12) converges.

THEOREM 11. The heat kernel of step $(m+1)$-Grushin operator from the origin is

$$
\mathcal{P}_{t}(x, y ; 0,0)=\frac{1}{t^{\alpha}} \int_{\mathbb{R}} e^{-\frac{f(x, y, 0, \lambda)}{t}} V(x, y, \lambda) d \lambda,
$$

where $f(x, y, 0, \lambda)$ is given in (3.50), and $V(x, y, \lambda)=W_{\lambda}(x, y, \lambda)$ with $W$ is a solution of the generalized transport equation (3.34) with $\alpha=m+\frac{1}{2}$.

In summary, we know that

(1) all the geodesics determine the geometry;

(2) all the information of the geodesics is contained in the complex action function $f$;

(3) one can then use $f$ to construct the fundamental solution and the heat kernel of $L_{G}$

(4) a through understanding of the action $f$ enables us to compute the small time asymptotics of the heat kernel at every critical point of $f$;

(5) the information contained in $f$ is revealed in these asymptotic expansions.

4. Heat kernels for operators $L_{j}, j=1,2,3,4$. For any positive integer $n$, consider the operator

$$
L_{1}=\frac{1}{2} \sum_{k=1}^{n}\left(\frac{\partial}{\partial x_{k}}\right)^{2}+\frac{1}{2} \sum_{k=1}^{n}\left(x_{k}^{m_{k}} \frac{\partial}{\partial y_{k}}\right)^{2},
$$

which can be rewritten as $L_{1}=\sum_{k=1}^{n} L_{G_{k}}$. For $k=1, \ldots, n, L_{G_{k}}$ is a step $m_{k}+1$ Grushin operator in $\left(x_{k}, y_{k}\right)$-plane. Since $\left[L_{G_{j}}, L_{G_{l}}\right]=0$ for $j \neq l$, it follows that

$$
e^{-t L_{1}}=e^{-t\left(L_{G_{1}}+\cdots+L_{G_{n}}\right)}=\prod_{k=1}^{n} e^{-t L_{G_{k}}} .
$$

In other words, the heat kernel is just the product of $n$ Grushin heat kernels.

The modified complex action function is the sum of $n$ 1-dimensional action functions. In particular,

$$
f(\mathbf{x}, \mathbf{y}, \mathbf{0}, \lambda)=\sum_{k=1}^{n} f_{k}\left(x_{k}, y_{k}, 0, \lambda_{k}\right),
$$

where $f_{k}$ has the form (3.50). The volume element is the product of $V_{k}, k=1, \ldots, n$ :

$$
V(\mathbf{x}, \mathbf{y}, \mathbf{0}, \lambda)=\prod_{k=1}^{n} V_{k}\left(x_{k}, y_{k}, 0, \lambda_{k}\right) .
$$

Therefore,

$$
\mathcal{P}_{t}(\mathbf{x}, \mathbf{y}, \mathbf{0}, \mathbf{0})=\frac{1}{(2 \pi t)^{m_{1}+\cdots+m_{n}+\frac{n}{2}}} \int_{\mathbb{R}^{n}} e^{-\frac{f(\mathbf{x}, \mathbf{y}, \mathbf{0}, \lambda)}{t}} V(\mathbf{x}, \mathbf{y}, \mathbf{0}, \lambda) d \lambda .
$$


In particular, if $m_{1}=\cdots=m_{n}=1$, the heat kernel can be written in an elegant form. In this case, the modified complex action function is

$$
f\left(\mathbf{x}, \mathbf{y}, \mathbf{x}_{0}, \lambda\right)=-i \sum_{k=1}^{n} \lambda_{k} y_{k}+\frac{\lambda_{k}}{4}\left[\left(x_{k}+x_{k 0}\right)^{2} \tanh \frac{\lambda_{k}}{2}+\left(x_{k}-x_{k 0}\right)^{2} \operatorname{coth} \frac{\lambda_{k}}{2}\right]
$$

The volume element is the product of $V_{k}\left(\lambda_{k}\right), k=1, \ldots, n: V(\lambda)=\prod_{k=1}^{n}\left(\frac{\lambda_{k}}{\sinh \lambda_{k}}\right)^{1 / 2}$. Therefore, one may recover the results in $[11,12]$ :

$$
\begin{aligned}
& \mathcal{P}_{t}\left(\mathbf{x}, \mathbf{y}, \mathbf{x}_{0}\right)=\frac{1}{(2 \pi t)^{\frac{3 n}{2}}} \int_{\mathbb{R}^{n}} e^{-\frac{f\left(\mathbf{x}, \mathbf{y}, \mathbf{x}_{0}, \lambda\right)}{t}} V(\lambda) d \tau \\
= & \frac{1}{(2 \pi t)^{\frac{3 n}{2}}} \int_{\mathbb{R}^{n}} e^{\frac{1}{t}\left\{i \sum_{k=1}^{n} \lambda_{k} y_{k}-\frac{\lambda_{k}}{4}\left[\left(x_{k}+x_{k 0}\right)^{2} \tanh \frac{\lambda_{k}}{2}+\left(x_{k}-x_{k 0}\right)^{2} \operatorname{coth} \frac{\lambda_{k}}{2}\right]\right\}} \prod_{k=1}^{n} V_{k}\left(\lambda_{k}\right) d \lambda .
\end{aligned}
$$

Now let us turn to the operator $L_{2}=\frac{1}{2}\left(\frac{\partial}{\partial x}\right)^{2}+\frac{1}{2} \sum_{k=1}^{n}\left(x^{m} \frac{\partial}{\partial y_{k}}\right)^{2}, m \in \mathbf{N}$. Since the $\sum_{k=1}^{n}\left(\frac{\partial}{\partial y_{k}}\right)^{2}$ is invariant under rotation, therefore, the modified complex action and volume element should depend on $|\vec{\lambda}|=\sqrt{\sum_{k=1}^{n} \lambda_{k}^{2}}$. Using the same method as we construct the heat kernel for the operator $L_{1}$, we know that:

$$
\mathcal{P}_{t}\left(x, \mathbf{y}, x_{0}\right)=\frac{1}{(2 \pi t)^{\frac{2 n+1}{2}}} \int_{\mathbb{R}^{n}} e^{-\frac{f\left(x, \mathbf{y}, x_{0}, \vec{\lambda}\right)}{t}} V(\vec{\lambda}) d \vec{\lambda},
$$

where the modified complex action function is

$$
f(x, \mathbf{y}, 0, \vec{\lambda})=\lambda\left[\frac{y}{x^{m-1}}\left|\mathcal{S}_{m}(|\vec{\lambda}|)\right|^{m-1}+\frac{x^{2}}{2(m+1)} \frac{(m-1)|\vec{\lambda}|+2 \mathcal{S}_{m}(|\vec{\lambda}|) \mathcal{S}_{m}^{\prime}(|\vec{\lambda}|)}{\mathcal{S}_{m}^{2}(|\vec{\lambda}|)}\right]
$$

In particular, $m=1$, one has

$$
f\left(x, \mathbf{y}, x_{0}, \vec{\lambda}\right)=-i \sum_{k=1}^{n} \lambda_{k} y_{k}+\frac{|\vec{\lambda}|}{2 \sinh |\vec{\lambda}|}\left(\left(x^{2}+x_{0}^{2}\right) \cosh |\vec{\lambda}|-2 x x_{0}\right),
$$

and the volume element is

$$
V(\vec{\lambda})=V\left(\lambda_{1}, \ldots, \lambda_{n}\right)=\left(\frac{|\vec{\lambda}|}{\sinh |\vec{\lambda}|}\right)^{1 / 2}
$$

Next, let us consider the operator $L_{3}=\frac{1}{2} \sum_{k=1}^{n}\left(\frac{\partial}{\partial x_{k}}\right)^{2}+\frac{1}{2} \sum_{k=1}^{n}\left(x_{k}^{m_{k}} \frac{\partial}{\partial y}\right)^{2}$, $m \in \mathbf{N}$. This operator can be written as $L_{3}=\sum_{k=1}^{n}\left(\widetilde{X}_{k}^{2}+\widetilde{Y}_{k}^{2}\right)=\sum_{k=1}^{n} L_{\widetilde{G}_{k}}$, where $\widetilde{X}_{k}=\frac{\partial}{\partial x_{k}}$ and $\widetilde{Y}_{k}=x_{k}^{m_{k}} \frac{\partial}{\partial y}$. Since $\left[L_{\widetilde{G}_{k}}, L_{\widetilde{G}_{j}}\right]=0$ for $k \neq j$. Hence,

$$
e^{-t L_{3}}=e^{-t\left(L_{\widetilde{G}_{1}}+\cdots+L_{\widetilde{G}_{n}}\right)}=\prod_{k=1}^{n} e^{-t L_{\widetilde{G}_{k}}} .
$$

In this case, the modified action function $f(\mathbf{x}, y, \mathbf{0}, \lambda)$ will be

$$
f(\mathbf{x}, y, \mathbf{0}, \lambda)=\lambda \sum_{k=1}^{n}\left[\frac{y}{x_{k}^{m_{k}-1}}\left|\mathcal{S}_{m_{k}}(\lambda)\right|^{m_{k}-1}+\frac{x_{k}^{2}}{2\left(m_{k}+1\right)} \frac{\left(m_{k}-1\right) \lambda+2 \mathcal{S}_{m_{k}}(\lambda) \mathcal{S}_{m_{k}}^{\prime}(\lambda)}{\mathcal{S}_{m_{k}}^{2}(\lambda)}\right] .
$$


In particular, $m_{k}=1$ for $k=1, \ldots, n$, one has

$$
\mathcal{P}_{t}\left(\mathbf{x}, y, \mathbf{x}_{0}\right)=\frac{1}{(2 \pi t)^{\frac{n+2}{2}}} \int_{\mathbb{R}} e^{-\frac{f\left(\mathbf{x}, y, \mathbf{x}_{0}, \lambda\right)}{t}} V(\lambda) d \lambda
$$

where the modified complex action function is

$$
f\left(\mathbf{x}, y, \mathbf{x}_{0}, \lambda\right)=-i \lambda y+\frac{\lambda}{2 \sinh \lambda}\left(\left(|\mathbf{x}|^{2}+\left|\mathbf{x}_{0}\right|^{2}\right) \cosh \lambda-2\left\langle\mathbf{x}, \mathbf{x}_{0}\right\rangle\right),
$$

and the volume element is $V(\lambda)=\left(\frac{\lambda}{\sinh \lambda}\right)^{n / 2}$ which is the solution of the transport equation

$$
\lambda \frac{\partial V}{\partial \lambda}+\left(\frac{n}{2} \lambda \operatorname{coth} \lambda+1-\alpha\right) V=0 \quad \text { with } \quad \alpha=\frac{n+2}{2} .
$$

Finally, let us turn to the operator $L_{4}=\frac{1}{2} \sum_{k=1}^{n+1}\left(\frac{\partial}{\partial x_{k}}\right)^{2}+\frac{1}{2} \sum_{k=1}^{n}\left(x_{k}^{m_{k}} \frac{\partial}{\partial y_{k}}\right)^{2}$. In fact, we may consider a more general operator:

$$
L_{4, n, \ell}=\frac{1}{2} \sum_{j=1}^{n} \frac{\partial^{2}}{\partial x_{j}^{2}}+\frac{1}{2} \sum_{j=1}^{n} x_{j}^{2 m_{j}} \frac{\partial^{2}}{\partial y_{j}^{2}}+\frac{1}{2} \sum_{k=n+1}^{n+\ell} \frac{\partial^{2}}{\partial x_{k}^{2}}=L_{1}+\Delta_{\ell},
$$

where $\Delta_{\ell}=\frac{1}{2} \sum_{k=n+1}^{n+\ell} \frac{\partial^{2}}{\partial x_{k}^{2}}$. Since $\left[L_{1}, \Delta_{\ell}\right]=0$, it follows that

$$
e^{-t L_{4, n, \ell}}=e^{-t\left(L_{1}+\Delta_{\ell}\right)}=e^{-t L_{1}} \cdot e^{-t \Delta_{\ell}} .
$$

We have already calculated the heat kernel for $L_{1}$ and the heat kernel for $\Delta_{\ell}$ is the $\ell$-dimensional Gaussian. Therefore, the heat kernel for the operator $L_{4, n, \ell}$ has the following form:

$$
\mathcal{P}_{t}\left(\mathbf{x}, \mathbf{y}, \mathbf{x}_{0}\right)=\frac{1}{(2 \pi t)^{\sum_{k=1}^{n} m_{k}+\frac{n+\ell}{2}}} \int_{\mathbb{R}^{n}} e^{-\frac{f\left(\mathbf{x}, \mathbf{x}_{0}, \mathbf{0}, \lambda\right)}{t}} V\left(\mathbf{x}, \mathbf{y}, \mathbf{x}_{0}, \lambda\right) d \lambda,
$$

where

$$
f\left(\mathbf{x}, \mathbf{y}, \mathbf{x}_{0}, \lambda\right)=\sum_{k=1}^{n} f_{k}\left(x_{k}, y_{k}, x_{k 0}, 0, \lambda_{k}\right)+\sum_{k=n+1}^{n+\ell} \frac{x_{k}^{2}}{2}
$$

and the volume element $V\left(\mathbf{x}, \mathbf{y}, \mathbf{x}_{0}, \lambda\right)=\prod_{k=1}^{n} V_{k}\left(x_{k}, y_{k}, x_{k 0}, \lambda_{k}\right)$.

In particular, $m_{1}=\cdots=m_{n}=1$, one has the volume element is $V(\lambda)=$ $\prod_{k=1}^{n}\left(\frac{\lambda_{k}}{\sinh \lambda_{k}}\right)^{1 / 2}$ and

$$
f\left(\mathbf{x}, \mathbf{y}, \mathbf{x}_{0}, \lambda\right)=-i \sum_{k=1}^{n} \lambda_{k} y_{k}+\frac{\lambda_{k}}{2 \sinh \lambda_{k}}\left[\left(x_{k}^{2}+x_{k 0}^{2}\right) \cosh \lambda_{k}-2 x_{k} x_{k 0}\right]+\sum_{k=n+1}^{n+\ell} \frac{x_{k}^{2}}{2}
$$

In this case, the heat kernel has the form:

$$
\mathcal{P}_{t}\left(\mathbf{x}, \mathbf{y}, \mathbf{x}_{0}\right)=\frac{1}{(2 \pi t)^{\frac{3 n+\ell}{2}}} \int_{\mathbb{R}^{n}} e^{-\frac{f\left(\mathbf{x}, \mathbf{y}, \mathbf{x}_{0}, \lambda\right)}{t}} V(\lambda) d \lambda .
$$


Acknowledgment. The final version of this paper is based on a talk given by the first author at the International Conference on Complex Geometry, Singularities and Related Fields in honor of the 60th birthday of Professor Stephen Yau which was held from August 3-6, 2012 at Tsinghua University, Beijing, China. The first author would like to thank the organizing committee for the invitation and the warm hospitality he has received from the Mathematical Sciences Center at Tsinghua University while he visited Beijing. Both authors would also like to thank Professor Ovidiu Calin for careful reading of the manuscript and many helpful suggestions.

\section{REFERENCES}

[1] J. V. Armitage and W. F. Eberlein, Elliptic functions, London Mathematical Society Student Texts, 67, Cambridge University Press, Cambridge, 2006.

[2] R. Beals, B. Gaveau, and P. C. Greiner, The green function of model two step hypoelliptic operators and the analysis of certain tangential Cauchy-Riemann complexes, Adv. Math., 121 (1996), pp. 288-345.

[3] R. Beals, B. Gaveau, and P. C. Greiner, On a geometric formula for the fundamental solutions of subelliptic Laplacians, Math. Nachr., 181 (1996), pp. 81-163.

[4] R. Beals, B. Gaveau, And P. C. Greiner, Hamilton-Jacobi theory and the heat kernel on the Heisenberg groups, J. Math. Pures Appl., $79: 7$ (2000), pp. 633-689.

[5] R. Beals, B. Gaveau, P. Greiner, and Y. Kannai, Exact fundamental solutions for a class of degenerate elliptic operators, Comm. Partial Differential Equations, 24 (1999), pp. 719742 .

[6] O. Calin and D. C. Chang, The geometry on a step 3 Grushin model, Applicable Analysis, $84: 2$ (2005), pp. 111-129.

[7] O. Calin And D. C. Chang, Sub-Riemannian Geometry: General Theory and Examples, Encyclopedia of Mathematics and Its Applications, 126, Cambridge University Press, Cambridge-New York, (2009).

[8] O. Calin, D. C. Chang, K. Furutani, and C. Iwasaki, Heat Kernels for Elliptic and Subelliptic Operators: Methods and Techniques, Birkhäuser-Verlag, Boston, 2010.

[9] O. Calin, D. C. Chang, and P. C. Greiner, Geometric Analysis on the Heisenberg Group and Its Generalizations, AMS/IP series in Advanced Mathematics, 40, American Mathematics Society and International Press, Cambridge, Massachusetts, (2007).

[10] O. Calin, D. C. Chang, P. C. Greiner, and Y. Kannai, On the geometry induced by a Grusin operator, Complex analysis and dynamical systems II, Contemporary Math., 382 (2005), pp. 89-111.

[11] O. Calin, D. C. Chang, J. S. Hu, And Y. Li, On the geometry induced by a class of degenerate elliptic operators, Journal of Nonlinear Analysis and Convex Analysis, 12 (2011), pp. 309340 .

[12] O. Calin, D. C. Chang, J. S. Hu, And Y. Li, Heat kernels for a class of degenerate elliptic operators using stochastic method, Complex Variables and Elliptic Equations, 57:2-4 (2012), pp. 155-168.

[13] C. H. Chang, D. C. Chang, B. Gaveau, P. Greiner, and H. P. Lee, Geometric analysis on a step 2 Grusin operator, Bull. Inst. Math., Academia Sinica, New Series, 4:2 (2009), pp. $119-188$.

[14] C. H. Chang, D. C. Chang, P. Greiner, and H. P. Lee, The positivity of the heat kernel on Heisenberg group, Anal. Appl., (singap), 11:5 (2013), pp. 1-16.

[15] D. C. Chang And Y. LI, SubRiemannian geodesics in Grushin plane, J. Geom. Anal., 22:3 (2012), pp. 800-826.

[16] W. L. CHOW, Uber systeme von linearen partiellen differetialgleichungen erster ordnug, Math. Ann., 117 (1939), pp. 98-105.

[17] E. A. Coddington, An Introduction to Ordinary Differential Equations, Dover publications Inc., New York, 1989.

[18] B. Gaveau, Principle de moindre action, propagation de la chaleur et estimeems sous elliptiques sur certains groups nilpotents, Acta. Math., 139 (1977), pp. 95-153.

[19] P. C. Greiner and O. Calin, On subRiemannian geodesics, Anal. Appl., (Singap.), 1:3 (2003), pp. 289-350.

[20] B. Gaveau and P. C. Greiner, On geodesics in subRiemannian geometry, Bull. Inst. Math. Academia Sinica (new series), 1:1 (2006), pp. 79-209. 
[21] B. Gaveau and P. C. Greiner, On geodesics in subRiemannian geometry II, Anal. Appl. (Singap.), 5:4 (2007), pp. 301-412.

[22] P. C. Greiner, On Hörmander operators and non-holonomic geometry, in: "Pseudodifferential operators: partial differential equations and time-frequency analysis", pp. 1-25, Fields Inst. Commun., 52, Amer. Math. Soc., Providence, RI, 2007.

[23] M. Gromov, Carnot-Carathéodory spaces seen from within, Prog. Math., 144, Birkhäuser, Basel, 1996.

[24] V. V. GRushin, On a class of hypoelliptic operators, Math. USSR Sbornik, 125 (1970), pp. 458476.

[25] L. Hörmander, Hypoelliptic second order differential equations, Acta. Math., 119 (1967), pp. $147-171$

[26] A. HulaniCKI, The distribution of energy in the Brownian motion in the Gaussian field and analytic-hypoellipticity of certain subelliptic operators on the Heisenberg group, Studia Math., 56 (1976), pp. 165-173.

[27] D. F. Lawden, Elliptic functions and applications, Applied Math. Sciences, 80, Springer-Verlag, Berlin-Heidelberg-New York, 1989.

[28] R. Strichartz, Sub-Riemnanian geometry, J. Differential Geom., 24:2 (1986), pp. 221-263. 
